\title{
Experimental investigation of fluid flow in horizontal pipes system of various cross-section geometries
}

\author{
E. Farsirotou, ${ }^{1, a}$, D. Kasiteropoulou ${ }^{1}$, and D. Stamatopoulou ${ }^{1}$ \\ ${ }^{1}$ Technological Educational Institute of Thessaly, Department of Civil Engineering T.E. Larissa, 41110 Larissa, Greece
}

\begin{abstract}
The current research work presents experiments of an essentially incompressible fluid flow in pipes. The experimental equipment consists of a horizontal pipe including a gate valve, a Venturi meter, a wide angle diffuser, an orifice plate, a 90-degree elbow and pressure tappings. An elbow connects the pipe to a rotameter with further pressure tappings. All pressure tappings connected to manometers held on a vertical panel behind the pipe work and show pressure at various points. The effect of the pipe geometry in the flow pattern is presented. Furthermore head losses are estimated, at specific stream-wise cross-sections, for mass flow rate numbered from 0.056 to $0.411 \mathrm{l} / \mathrm{s}$. The manometers measure and clearly show pressure distribution against a calibrated scale. The diagrams of mass flow rate and head losses are presented in specific crosssections, where geometry changes. All measurements were calibrated and validated in a maximum standard deviation difference of 5\%. The head losses decrease as the mass flow rate decreases, for all pipe geometries. In the future the experimental results can be used to verify numerical simulation results.
\end{abstract}

\section{Introduction}

The measurement of fluid flow is important in many applications ranging from blood-flow rates detection in human artery to the measurement of liquid oxygen in a rocket. Flow-rate-measurement devices frequently require accurate pressure, energy and temperature determination in order to calculate the output of the instrument. The most widely used flow metering principle involves placing a fixed area flow restriction of some type in the pipe or duct carrying the fluid. This flow restriction causes a pressure drop that varies with the flow rate. Thus, measurement of the pressure drop by means of a suitable differential-pressure pick up allows flow rate measurement.

Some of these recent applications and also other, which involve pressure drop on pipe, ducts or planar channels with grooved elements on one or both sides for Newtonian and Non-Newtonian fluids are briefly mentioned. U-Kaew [1] investigated of viscoelastic flow in a horizontal circular pipe and viscoelastic flow passing elbow geometry. He found that for Deborah numbers, $D e$, chosen between 0.59 and 5.3, the flow exhibits characteristics that can be found Newtonian fluid flow where differential pressure measurements between upstream and downstream of the test section are directly proportional to the flow rate. On the other hand, the elastic flow feature known as "die swell" was observed at the free surface of exit of the test channel during the experiment. Metwally [2] presented a review of compressible pulsating flow effects on system performance. In the application of an orifice plate experimental set-up showed that the resonance in the connecting leads can produce confusing effects when the lead lengths are not short compared with the quarterwavelength of any pulsation present. There are numerous difficulties in measuring pulsating differential pressures. The pulsation error due to the basic non-linearity of the pressure differential flow meter arises when a slowresponse differential sensor is used. Fester [3] presented a review of the loss coefficient data for laminar and turbulent flow of non-Newtonian fluids in pipe fittings. The loss coefficients in laminar flow for all fittings are orders of magnitude greater than those in turbulent flow. The purely viscous driven flow regime is primarily at $R e$ $<10$, after which turbulence will be introduced that is dependent on the geometry of the fitting. For some fittings, the minimum values obtained are very pronounced, especially for larger orifice ratios and larger valve sizes. For sudden contractions and long orifices, a smoother transition is observed. Eltoukhy [4] investigated the behavior of sand-water (two-phase) flows in inclined pipes. The pipe inclination angle was varied from $0^{\circ}$ to $90^{\circ}$ in upward and downward directions and the sand concentration in water was regulated up to $15 \%$ by volume. It was concluded that the head losses of the downward sloping pipe flow are always lower than the head losses of the horizontal flow and these are always lower than the head losses of the upward sloping pipe

\footnotetext{
$\overline{{ }^{a}}$ Corresponding author: efars@teilar.gr
} 
flow, regardless of the concentration and inclination angle.

Work has also been reported on experimental investigations in grooved flow geometries. Greiner [5] investigated the water flow through ducts with periodically grooved elements located into one wall. He found that while the absolute level of transport depends on the thermal and geometric conditions, the enhancement gained from $20 \%$ flow rate modulation is fairly insensitive to them. Herman and Kang [6] visualized the unsteady temperature fields in a grooved channel with curved vanes using interferometry and found a heat transfer enhance by a factor of 1.5-3.5, when compared to the basic grooved channel, mainly due to increased flow velocities in the groove region. Stroock et al. [7] investigated steady pressure-driven flows in microchannels, at low Reynolds numbers, and found that the hydrodynamic dispersion along the channel is reduced relative to the channel with flat walls.

In the present work experiments of an essentially incompressible fluid flow in pipes are presented. The experimental equipment consists of a horizontal pipe including a gate valve, a Venturi meter, a wide angle diffuser, an orifice plate, a 90-degree elbow and pressure tappings. The effect of the pipe geometry in the flow pattern is investigated. Moreover head losses are estimated, at specific stream-wise cross-sections, for mass flow rate numbered from 0.056 to $0.411 \mathrm{l} / \mathrm{s}$.

The current paper consists of the experimental set-up and the mathematical model presented in the section 2 . Experimental laboratory results are shown and discussed in section 3, whereas section 4 contains concluding remarks.

\section{Experimental set-up}

\subsection{Testing flume description and Experimental procedure}

The experimental study was conducted at the Hydraulics Laboratory, of Civil Engineering T.E. Larissa Department of the Technological Educational Institute of Thessaly. The used experimental laboratory equipment is presented in Figure 1.

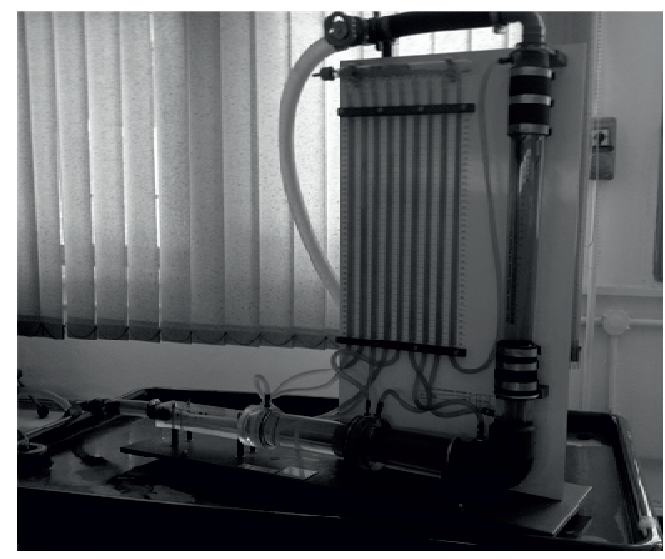

Figure 1. Presentation of the experimental laboratory equipment.
In the experimental set-up water from the hydraulic bench enters the horizontal circular pipe through a venturi meter, which consists of a gradually converging section, followed by a throat, and a long gradually diverging section. Next the flow continues through a rapidly diverging section, along a settling length and through an orifice plate meter. Furthermore the flow follows a further settling length, a right-angled bend and enters to a rotameter. This consists of a transparent tube in which a float takes up an equilibrium position. The position of this float is a measure of the flow rate. After the rotameter the water returns via a control valve to the hydraulic bench and the weigh tank. The laboratory equipment has nine pressure tappings each of which is connected to its own manometer, held on a vertical panel behind the pipe work for immediate read out of pressure values. A detailed diagram of the flow measuring experimental equipment with the diameters of each section of the circular pipe is given in Figure 2.

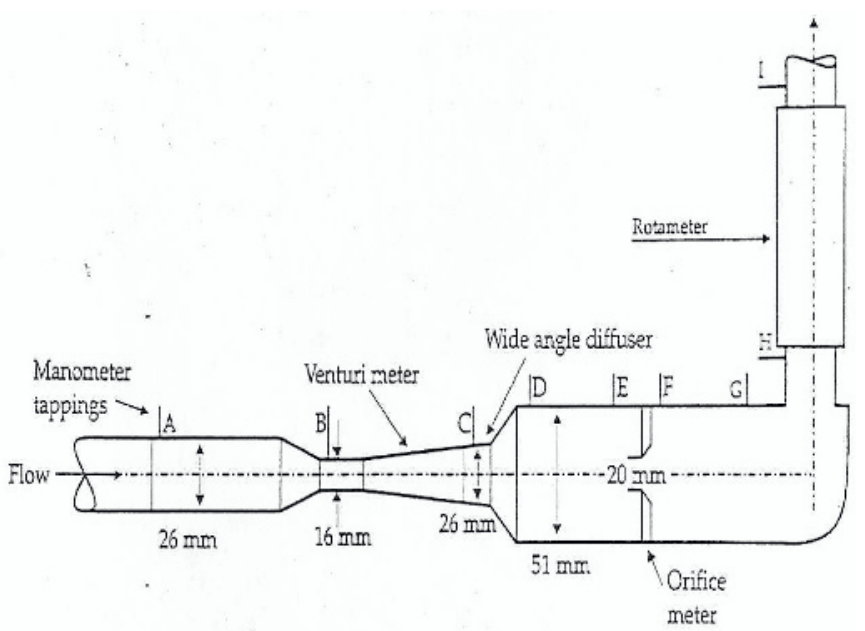

Figure 2. Detailed diagram of the flow measuring experimental equipment [8].

The study includes circular straight pipe and elbow geometries. The geometrical data of the tested experimental circular transparent Plexiglas pipe which was employed to study the viscoelastic flow characteristics are presented in Table 1. The pipe was placed horizontally on the hydraulic table and the flow system is divided into two components, the upstream pipe and the test section. The flow is able to reach the fully developed steady conditions with minimal influence of any upstream disturbances from elbows and valves. For the test section, flush terminals were mounted at various locations [8].

The standard $90^{\circ}(51.00 \mathrm{~mm})$ elbow with the radius of curvature, was also employed to study the viscoelastic effect on the flow structures. The elbow geometry system is designed to have a smooth surface inside preventing the disturbances from all sources such as threads and elbow connections. Flush mounted terminals were employed at the two locations at both ends of the elbow. The flow loop is sets as follows: Compressed air is applied to the storage tank to drive the working fluid through the horizontal test channel, while the pressure regulator maintains the constant input pressure level to ensure the constant flow rate. Pressure variation due to 
changes of the fluid level in the tank is negligible [9]. All experiments are conducted at the room temperature $(25$ $\left.{ }^{\mathrm{o}} \mathrm{C}\right)$.

Table 1. Geometrical data of the tested experimental pipe.

\begin{tabular}{|l|l|l|}
\hline $\mathrm{a} / \mathrm{a}$ & Cross-section diameter $\mathrm{D}(\mathrm{m})$ & Length $(\mathrm{m})$ \\
\hline 1 & 0.026 & 0.043 \\
\hline 2 & $\begin{array}{l}\text { gradually changed diameter } \\
\text { from 0.026m to } 0.016 \mathrm{~m}\end{array}$ & 0.024 \\
\hline 3 & 0.016 & 0.018 \\
\hline 4 & $\begin{array}{l}\text { gradually changed diameter } \\
\text { from 0.016m to 0.026m }\end{array}$ & 0.09 \\
\hline 5 & 0.026 & 0.036 \\
\hline 6 & $\begin{array}{l}\text { rapidly changed diameter from } \\
0.026 \mathrm{~m} \text { to } 0.051 \mathrm{~m}\end{array}$ & 0.026 \\
\hline 7 & 0.051 & 0.22 \\
\hline 8 & 0.020 & - \\
\hline 9 & 0.051 & 0.26 \\
\hline 10 & diameter at point $\mathrm{H} 0.019 \mathrm{~m}$ & - \\
\hline
\end{tabular}

In Figure 3 the areas of friction and minor losses are distinguished. There are five areas where friction head losses are developed, $h_{f 1}, h_{f 2}, h_{f 3}, h_{f 4}$ and $h_{f 5}$ and the minor head losses are developed in the abrupt changes of the cross-section geometry and are mentioned as $h_{m A B}, h_{m B C}$, $h_{m C D}, h_{m E F}$ and $h_{m G H}$.

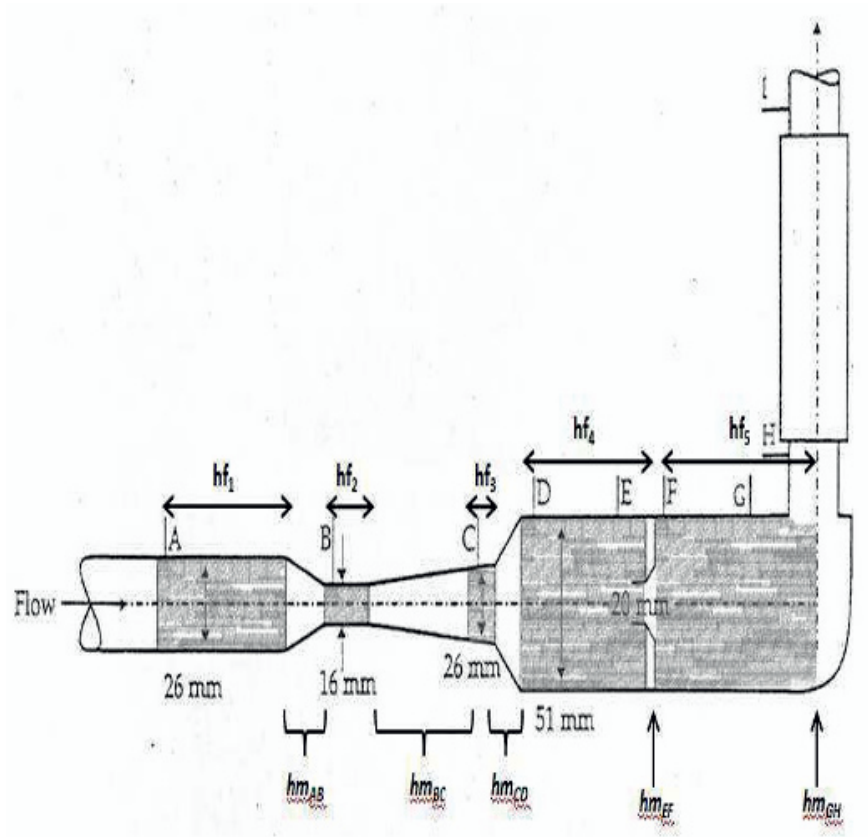

Figure 3. Detailed diagram of the flow friction and minor head losses along the measuring laboratory equipment.

\subsection{Equations and Mathematics}

For all experimental results water energy is computed from one cross section to the next by solving the Energy equation to a body of water enclosed by two cross sections at locations 1 and 2 as: $\frac{p_{1}}{p g}+z_{1}+\frac{V_{1}^{2}}{2 g}=\frac{p_{2}}{p g}+z_{2}+\frac{V_{2}^{2}}{2 g}+h_{\text {ftotal }}+h_{\text {mtotal }}(1)$

where:

$p$ is the pressure of the fluid at each cross-section, $\rho$ is the density of the fluid,

$g$ is the acceleration due to gravity,

$V$ is the average velocity at each cross-section,

$z$ is the pipe bottom elevation above a reference plane at each cross-section,

$h_{\text {ftotal }}$ are the total friction losses along the channel and $h_{m t o t a l}$ are the total minor losses along the channel.

The velocity and the pressure are calculated at each cross-section of the experimental fluid flow. Velocity is calculated based on the law of mass conservation whereas the pressure is measured in a manometer.

The total head loss is also presented. Total head loss is the combination of different types of losses mentioned as: friction losses and minor losses. The friction loss is given by the Darcy-Weisbach [10] equation, relates the head loss or pressure loss due to friction along a given length of pipe to the average velocity of the fluid flow and is given by the form:

$$
h_{f}=f \cdot \frac{L}{D} \cdot \frac{V^{2}}{2 g}
$$

where:

$f$ is the friction factor,

$L$ is the length of each part of the pipe and $D$ is the pipe diameter.

The minor head losses are calculated at all pipe geometries. The measurements of the minor head losses are compared to theoretical calculations expressed as:

$$
h_{m}=k \cdot \frac{V^{2}}{2 g}
$$

where:

$k$ is the minor loss coefficient depending on the pipe components [11].

\section{Analysis of experimental results}

Total head lines along the channel apparatus are presented in Figure 4, for all mass flow rate cases investigated in this work. As it is expected higher head losses are observed at higher mass flow rate values. This behaviour is more pronounced at the first and fourth cross-sections. The last probably happens due to the small length of these sections along the channel which causes detachment in the flow pattern and consequently significant high local energy losses.

Experimental measurements of minor head losses for mass flow rate numbered from 0.058 to $0.4111 / \mathrm{s}$, for each cross-section, are given in Table 2. All measurements were calibrated and validated in a maximum standard deviation difference of $5 \%$. Experimental measurements 
of minor head losses variation for mass flow rate numbered from 0.058 to $0.4111 / \mathrm{s}$, at three different crosssections: a. gradually diverging section (B-C), b. orifice plate meter (E-F) and c. $90^{\circ}$ elbow section $(\mathrm{G}-\mathrm{H})$, are presented in Figure 5. It turns out that minor head losses increase as the mass flow rate increases for all cross sections. This increase is more pronounced at steep crosssections differences.

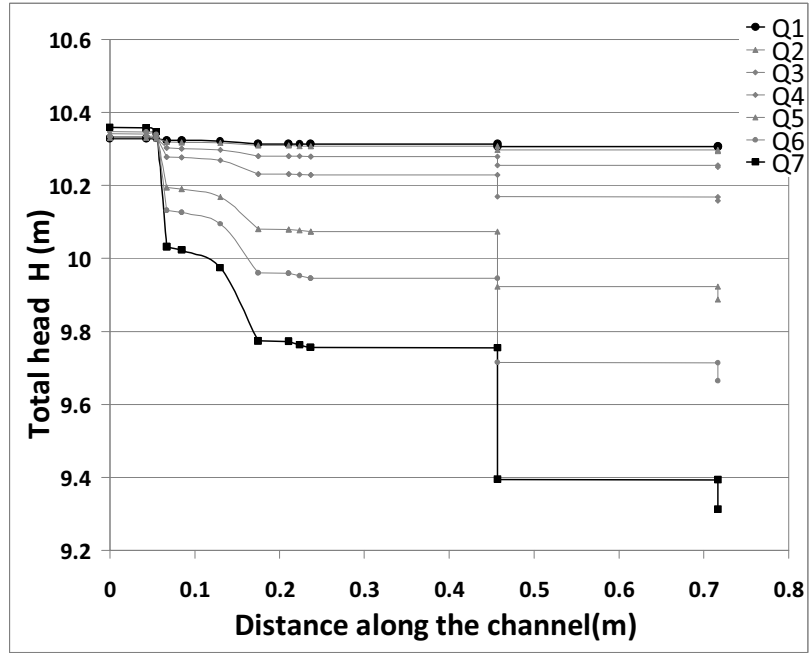

Figure 4. Total head lines along the channel apparatus for all mass flow rate cases: a. Q1 $=0.0581 / \mathrm{s}, \mathrm{b} . \mathrm{Q} 2=0.0791 / \mathrm{s}, \mathrm{c} . \mathrm{Q} 3=$ $0.1211 / \mathrm{s}, \mathrm{d} . \mathrm{Q} 4=0.1691 / \mathrm{s}$, e. $\mathrm{Q} 5=0.2761 / \mathrm{s}, \mathrm{f} . \mathrm{Q} 6=0.3271 / \mathrm{s}$ and g. Q7 $=0.4111 / \mathrm{s}$

Table 2. Experimental measurements of minor head losses for mass flow rate numbered from 0.058 to $0.411 \mathrm{l} / \mathrm{s}$.

\begin{tabular}{|c|c|c|c|c|c|c|c|c|}
\hline & Section & Q1 & Q2 & Q3 & Q4 & Q5 & Q6 & Q7 \\
\hline $\mathbf{1}$ & A-B & 0.009 & 0.011 & 0.029 & 0.055 & 0.147 & 0.215 & 0.327 \\
\hline $\mathbf{2}$ & B-C & 0.006 & 0.009 & 0.020 & 0.045 & 0.110 & 0.167 & 0.249 \\
\hline $\mathbf{3}$ & C-D & 0.001 & 0.001 & 0.001 & 0.003 & 0.006 & 0.013 & 0.017 \\
\hline $\mathbf{4}$ & E-F & 0.006 & 0.011 & 0.025 & 0.059 & 0.15 & 0.231 & 0.361 \\
\hline $\mathbf{5}$ & G-H & 0.001 & 0.003 & 0.005 & 0.012 & 0.036 & 0.050 & 0.082 \\
\hline
\end{tabular}

Minor head losses variation along the channel apparatus for all mass flow rate cases is presented in Figure 6. We observe that minor head losses for all mass flow rate values at the first and fourth cross section is higher. This behavior is in agreement with the headlines presented in Figure 4 and can be explained by the small apparatus length in these sections. On the other hand in the rapidly diverging section (C-D) minor head losses are lower than in all other cases and this can be explained by the lower velocities due to higher dimensions of the pipe diameter in this cross-section (abrupt change starting from $26 \mathrm{~mm}$ and reaching $51 \mathrm{~mm}$ ).

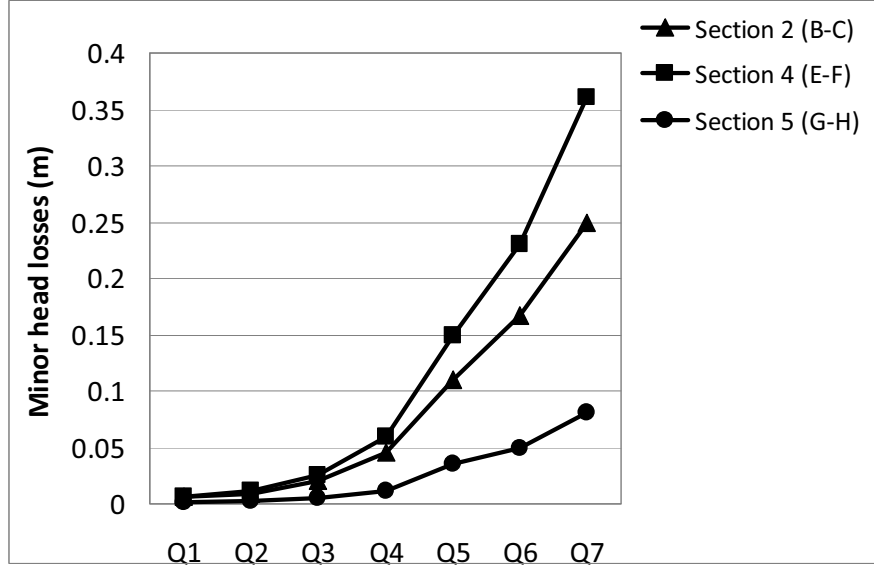

Figure 5. Experimental measurements of minor head losses variation at three different cross-sections: a. gradually diverging section (B-C), b. orifice plate meter (E-F) and c. $90^{\circ}$ elbow section $(\mathrm{G}-\mathrm{H})$.

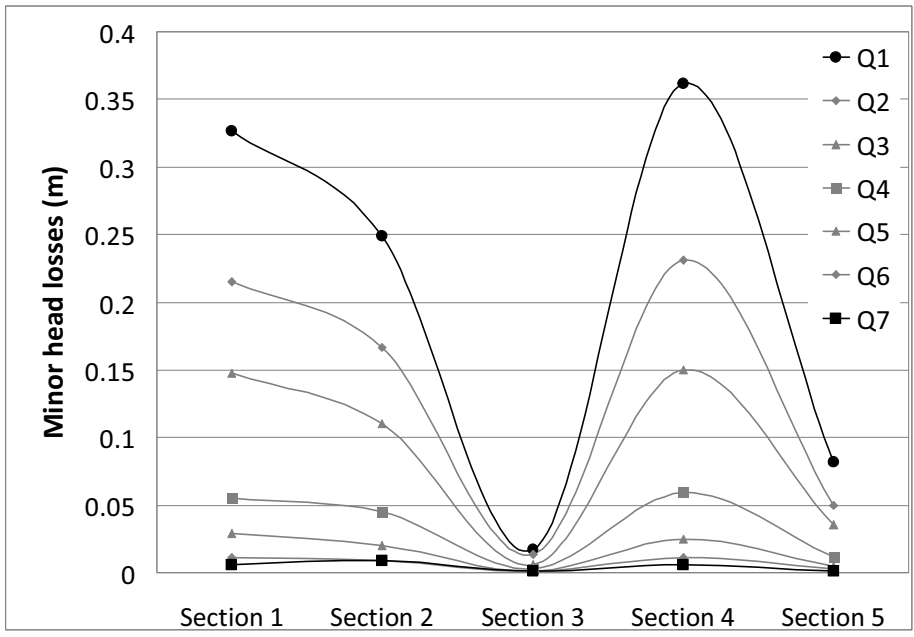

Figure 6. Experimental measurements of minor head losses variation along the channel apparatus for all mass flow rate cases: a. Q1 $=0.0581 / \mathrm{s}, \mathrm{b} . \mathrm{Q} 2=0.079 / \mathrm{s}, \mathrm{c} . \mathrm{Q} 3=0.1211 / \mathrm{s}, \mathrm{d} . \mathrm{Q} 4=$ $0.1691 / \mathrm{s}$, e. $\mathrm{Q} 5=0.2761 / \mathrm{s}, \mathrm{f} . \mathrm{Q} 6=0.3271 / \mathrm{s}$ and g. Q7 $=0.4111 / \mathrm{s}$

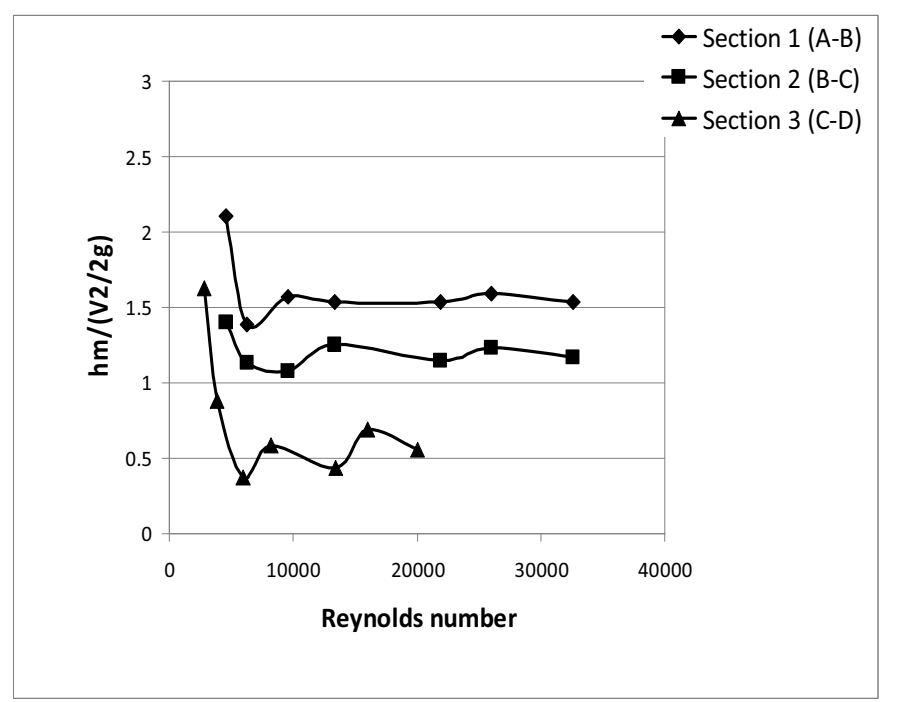

Figure 7. Non-dimensional minor head loss variation versus Reynolds number at three typical different cross-sections: a. gradually converging section (A-B), b. gradually diverging section (B-C) and c. gradually diverging section (C-D). 
The calculated non-dimensional minor head loss variation versus Reynolds number diagram, at three typical different cross-sections, is presented in Figure 7. For all cross-sections presented here the non-dimensional head loss decreases as Reynolds number increases, in the range of $2500 \leq R e \leq 5000$, whereas for higher Reynolds number the non-dimensional minor head loss remains almost constant within statistical errors.

\section{Conclusions}

Experiments of an essentially incompressible fluid flow in horizontal circular pipes system of different crosssection diameters are graphically presented. The key characteristics of the experimental results are discussed. Higher minor head losses are observed at higher mass flow rate values and the increment is more pronounced at abrupt cross-section changes. The experimental results are applicable in the development of new and the refinement of existing codes for computing head losses variation in horizontal circular pipes system of various cross-section geometries. Moreover, future research and development contains verification of three-dimensional incompressible fluid flow in planar channels, as well as development of existing ones.

\section{References}

1. A.U. Kaew, Lehigh University, Theses and Dissertations, Paper 829 (2003)

2. M. Metwally, 13th International Conference on ASAT- 13, May 26 - 28 (2009)

3. V. Fester, P. Slatter and N. Alderman, Rheology, Dr. Juan De Vicente (Ed.), (2012)

4. M.A.R. Eltoukhy, Int. J. Civil Eng Tech 4(3), 45-56, (2013)

5. M. Greiner, Int. J. Heat Mass Transfer 34(6), 13831391 (1990)

6. C. Herman and E. Kang, Int. J. Heat Mass Transfer 45, 3741-3757 (2002)

7. A.D. Stroock, S.K.W. Dertinger, A. Ajdari, I. Mezic, H.A. Stone, G.M. Whitesides, Science 295 , 647651 (2002)

8. http//www.tecquipment.com/Fluid-Mechanics/FlowPressure/H10.aspx.

9. H. Kim, A. Oztekin and S. Neti, J. Non-Newtonian Fluid Mech, 90, 261-281 (2000).

10. J. Weisbach, Lehrbuch der Ingenieur- und Maschinen-Mechanik, 1, Theoretische Mechanik, Vieweg und Sohn, Braunschweig. 535 pages (in German).

11. A. Liakopoulos, Hydraulics, Tziolas Publications (in Greek) (2013) 